\title{
Detecting the spin-orbit misalignment of the super-Earth 55 Cancri $e^{\star}$
}

\author{
Vincent Bourrier ${ }^{1}$ and Guillaume Hébrard ${ }^{1,2}$ \\ ${ }^{1}$ Institut d'astrophysique de Paris, UMR 7095 CNRS, Université Pierre \& Marie Curie, 98bis boulevard Arago, 75014 Paris, France \\ e-mail: bourrier@iap.fr \\ 2 Observatoire de Haute-Provence, CNRS/OAMP, 04870 Saint-Michel-l’Observatoire, France
}

Received 24 May 2014 / Accepted 25 June 2014

ABSTRACT

\begin{abstract}
We present time-resolved spectroscopy of transits of the super-Earth 55 Cnc e using HARPS-N observations. We devised an empirical correction for the "color effect" on the radial velocity residuals from the Keplerian fit, which significantly improves their dispersion with respect to the HARPS-N pipeline standard data reduction. Using our correction, we were able to detect the smallest RossiterMcLaughlin anomaly amplitude of an exoplanet so far $(\sim 60 \mathrm{~cm} / \mathrm{s})$. The super-Earth $55 \mathrm{Cnc}$ e is also the smallest exoplanet with a Rossiter-McLaughlin anomaly detection. We measured the sky-projected obliquity $\lambda=72.4_{-11.5}^{+12.0^{\circ}}$, indicating that the planet orbit is prograde, highly misaligned and nearly polar compared to the stellar equator. The entire 55 Cancri system may have been highly tilted by the presence of a stellar companion.
\end{abstract}

Key words. Planets and satellites: dynamical evolution and stability - techniques: radial velocities - stars: individual: 55 Cancri

\section{Introduction}

Spectroscopic observations during the transit of an exoplanet across its host star can measure the sky-projected angle between the spins of the planetary orbit and the stellar rotation (namely the obliquity) through the Rossiter-McLaughlin (RM) effect (Holt 1893; Rossiter 1924; McLaughlin 1924). The occultation of a rotating star by a planet distorts the apparent stellar line shape by removing the profile part emitted by the hidden portion of the star. This induces anomalous stellar radial velocity variations during the transit, which constrain the skyprojected obliquity $(\lambda)$. Whereas first observed systems revealed aligned, prograde orbits (e.g., Queloz et al. 2000; Winn et al. 2005; Loeillet et al. 2008), first misaligned systems were reported with the cases of XO-3 (Hébrard et al. 2008; Winn et al. 2009c; Hirano et al. 2011) and HD 80606 (Moutou et al. 2009; Pont et al. 2009; Winn et al. 2009a; Hébrard et al. 2010). About thirty misaligned systems have been identified today over more than eighty measured systems ${ }^{1}$ (Albrecht et al. 2012; Crida \& Batygin 2014), including some with retrograde or nearly polar orbits (e.g., Winn et al. 2009b; Narita et al. 2010; Triaud et al. 2010; Hébrard et al. 2011). These unexpected results favor scenarios where close-in massive planets have been brought in by planet-planet (or planet-star) scattering, Kozai migration, and/or tidal friction, rather than more standard scenarios implying disk migration that are expected to conserve the initial alignment between the angular momentums of the disk and of the planetary orbits (see, e.g., Fabrycky \& Tremaine 2007; Guillochon et al. 2011), although some models show that the initial misalignment of a planet can be maintained through its interactions with the disk (Teyssandier et al. 2013). Alternatively, it has been proposed that the orbit still reflects the orientation of the disk,

\footnotetext{
* Table 4 is available in electronic form at http://www . aanda.org 1 The Holt-Rossiter-McLaughlin Encyclopaedia: http://www . physics.mcmaster.ca/ rheller/
}

with the stellar spin instead having moved away, either earlyon through magnetosphere-disk interactions (Lai et al. 2011) or later through elliptical tidal instability (Cébron et al. 2011).

Obliquity measurements have mainly been done in singleplanet systems, mostly on hot-Jupiters. In recent years, they have been extended to transiting multiplanet systems, most of which host super-Earths. Obliquities have been derived from the RM anomaly in the systems KOI-94 (Hirano et al. 2012; Albrecht et al. 2013) and Kepler-25 (Albrecht et al. 2013), from starspot variations of Kepler-30 (Sanchis-Ojeda et al. 2012), and from the measure of the stellar inclination via asteroseismology in the sytems Kepler-50 and Kepler-65 (Chaplin et al. 2013), and the system Kepler-410 (Van Eylen et al. 2014). These systems have shown coplanar orbits that are well aligned with the stellar equator, hinting that their orbital planes still trace the primordial alignment of the protoplanetary disk responsible for the planets' migrations, while the apparent isotropic distribution of obliquities of hot-Jupiters is the result of dynamical interactions (Albrecht et al. 2013). This conclusion has recently been put in doubt by the large obliquity of the two-planet system Kepler-56 (Huber et al. 2013) and the possible spin-orbit misalignements of several multicandidate Kepler systems (Walkowicz \& Basri 2013; Hirano et al. 2014).

The exoplanet 55 Cnc e offers the opportunity to probe spinorbit misalignments in the domains of both multiple systems and super-Earths. It is part of a five-planet system, which was first detected and characterized with radial velocity measurements (Fischer et al. 2008). The orbital period of the closest and lightest of them, planet $55 \mathrm{Cnce}$, was a subject of debate because of the aliasing in the radial velocity datasets. The value $P=0.7365$ days proposed by Dawson \& Fabrycky (2010) was confirmed when Winn et al. (2011) with MOST and Demory et al. (2011) with Warm Spitzer, detected photometric transits of planet $\mathrm{e}$ at the ephemeris corresponding to that short period. Subsequent studies have refined the orbital and transit 
Table 1. Log of the HARPS-N observations.

\begin{tabular}{lccccccc}
\hline \hline Run & Transit mid-time (UT) & Exposures $^{\dagger}$ & Mode $^{*}$ & Airmass & S/N_392* & S/N_527* & S/N_673* \\
\hline A & $2012-12-26$ at $02 \mathrm{~h} 54$ & 27 & ThAr $^{* *}$ & $1.10-1.00-1.35$ & 56 & 261 & 150 \\
$\mathrm{~B}$ & $2014-01-02$ at $01 \mathrm{~h} 48$ & 33 & FP & $1.00-1.43$ & 70 & 342 & 260 \\
$\mathrm{C}$ & $2014-01-27$ at $02 \mathrm{~h} 49$ & 30 & FP & $1.00-1.38$ & 27 & 144 & 131 \\
$\mathrm{D}$ & $2014-02-27$ at $01 \mathrm{~h} 15$ & 30 & ThAr & $1.00-1.39$ & 16 & 92 & 93 \\
$\mathrm{E}$ & $2014-03-29$ at $23 \mathrm{~h} 41$ & 27 & FP & $1.01-1.36$ & 73 & 354 & 269 \\
\hline
\end{tabular}

Notes. ${ }^{(\dagger)}$ Number of 6-min individual exposures. ${ }^{(\ddagger)}$ Simultaneous thorium-argon (ThAr) or Fabry Perot (FP) reference. ${ }^{(\dagger \dagger)}$ Airmass evolution during the observation sequence. ${ }^{(*)}$ Median of the signal-to-noise ratio per pixel at $392 \mathrm{~nm}$ (pipeline order \# 2), $527 \mathrm{~nm}$ (\#42), and 673 nm (\#67). ${ }^{(* *)}$ Except for the two first exposures made without simultaneous reference.

parameters of $55 \mathrm{Cnc}$ e using additional photometry and radial velocity measurements (e.g., Demory et al. 2012; Gillon et al. 2012; Endl et al. 2012). Thus, 55 Cnce is an unusually close-in $(a=0.015 \mathrm{au})$ super-Earth with a mass $M_{\mathrm{p}}=7.99 \pm 0.25 M_{\oplus}$ (Nelson et al. 2014) and a radius $R_{\mathrm{p}}=1.99 \pm 0.08 R_{\oplus}$ in the optical (Dragomir et al. 2014).

$55 \mathrm{Cnc}$ is the only naked-eye star hosting a transiting planet. The brightness of that nearby G8V $\operatorname{star}(V=5.95, d=12.3 \mathrm{pc})$ makes it a particularly advantageous target for follow-up studies. In particular, it allowed the brightness temperature measurement of $55 \mathrm{Cnc}$ e $(T=2360 \pm 300 \mathrm{~K})$ thanks to Spitzer observation of occultations (Demory et al. 2012), as well as the possible detection of $\mathrm{HI}$ in the atmosphere of $55 \mathrm{Cncb}(P=14.652$ days, $M_{\mathrm{p}}=7.8 \pm 0.6 M_{\oplus}$ ) with HST (Ehrenreich et al. 2012), indicating that this planet might host an extended atmosphere and suggesting that the orbits of all the planets of the system are nearly coplanar. The orbital evolution of this closely packed system has motivated several studies that also point toward a coplanar and dynamically stable system (Nelson et al. 2014; Kaib et al. 2011; Boué \& Fabrycky 2014). Kaib et al. (2011) show that the 55 Cnc system should be highly misaligned with a true obliquity of $\sim 65^{\circ}$, while Boué \& Fabrycky (2014) point out that this requires the stellar spin axis to be weakly coupled to the planets' orbits. Although the detection of the RM anomaly of planet e is expected to be challenging (amplitude $<1 \mathrm{~m} / \mathrm{s}$ ), its short period, small radius, and its part in a complex multiple system makes it a particularly interesting target for investigating misalignment.

We describe in Sect. 2 the observations made with the HARPS-N spectrograph. In Sect. 3 we describe the color effect and its correction, in Sect. 4 we present the detection of the Rossiter-McLaughlin anomaly, and in Sect. 5 we test its robustness. Discussion of the results will be found in Sect. 6 .

\section{Observations and data reduction}

We obtained time to observe a total of eight different transits of $55 \mathrm{Cnce}$ over three different semesters between late 2012 and early 2014 with the spectrograph HARPS-N at the 3.58-m Telescopio Nazionale Galileo (TNG, La Palma, Spain). HARPS-N is a fiber-fed, cross-dispersed, environmentally stabilized echelle spectrograph dedicated to high-precision radial velocity measurements (Cosentino et al. 2012). It provides the resolution power $\lambda / \Delta \lambda=115000$. The light is dispersed on 69 spectral orders from 383 to $690 \mathrm{~nm}$. Due to weather and technical problems, three of the eight scheduled transits could not be observed at all. The log of the five observed transits (runs hereafter labeled from A to E) is reported in Table 1. Runs A to $\mathrm{D}$ were executed in service mode by the TNG Team, whereas Run E was made in visiting mode by us.

All the observations were sequences of about thirty successive exposures of six-minute durations each. We chose that duration as a compromise between accuracy, temporal resolution, and overheads. Each sequence lasts several hours (about three hours typically, whereas the full transit lasts $1.5 \mathrm{~h}$ ). For technical reasons, a poor coverage of the transit was obtained during Run A, with only six measurements secured during the transit itself. The four other runs allowed good coverage of the whole transit duration to be obtained. Reference observations were secured immediately before and after the transit for Runs B, D, and E; for Runs A and C, those reference observations were secured mainly after the transit. The observations of Run E had to be stopped earlier than scheduled due to a sudden degradation of the weather conditions after the end of the transit.

The CCD was used in its fast readout mode with a speed of $500 \mathrm{kHz}$. We used the two 1"-wide optical-fiber apertures: the first one was on the target, whereas the second one was used for simultaneous radial-velocity reference, using the thorium-argon lamp or the Fabry Perot depending of the run (see Table 1). The two first exposures of Run A are an exception, since they were observed without simultaneous reference, the second aperture being on the nearby sky.

Owing to different weather conditions (seeing and absorption), the signal-to-noise ratios ( $/ \mathrm{Ns}$ ) were different among the five runs. Table 1 reports typical $\mathrm{S} / \mathrm{Ns}$ for each run in three different parts of the spectra: Runs C and D were obtained in poor conditions and provide data of relatively low accuracy, Runs B and $\mathrm{E}$ were obtained in good conditions and provide particularly high-accuracy data, whereas Run A is intermediate.

The $\mathrm{S} / \mathrm{N}$ values reported in Table 1 are the median among the $\sim 30$ exposures of a given run in a given spectral order. In fact, the $\mathrm{S} / \mathrm{N}$ is varying significantly with time during a given run, and these variations are of different amplitudes from one spectral order to the other. This means that there is a global variation in the flux during a run, but also a variation in the distribution of the flux with the color (hereafter named "color effect"). The global and chromatic S/N variations show random structures on different time scales, which are probably mainly due to shortterm variations in the weather conditions. They also show regular, lower-frequency variations that could be explained by the airmass change of the target during each run, which translates into wavelength-dependent throughput variations. The airmass evolution during each run is reported in Table 1 . The airmass monotonously varies for all the runs but the first one. In the case of Run A, the target reached the meridian during the sequence; it implied an interruption of the observations after the first seven exposures, then a change in the orientation of the alt-azimuth TNG telescope before starting the observations again. The few radial velocities obtained during transit $A$ were thus secured in different conditions than the reference ones obtained after that transit. Runs B to E were fully executed after the target reached the meridian. 
The HARPS-N spectra were extracted from the detector images with the DRS pipeline, which includes localization of the spectral orders on the 2D-images, optimal order extraction, cosmic-ray rejection, corrections of flat-field, wavelength calibration with thorium-argon lamp exposures made during the afternoon, and short-term radial-velocity drift correction from simultaneous references with thorium-argon or Fabry Perot. Then the spectra passed through weighted cross-correlation with a G2-type numerical masks following the method described by Baranne et al. (1996) and Pepe et al. (2002). All the exposures provide a well-defined, single peak in the cross-correlation function (CCF), whose Gaussian fits allow the radial velocities to be measured, together with their associated uncertainties. We tested different kinds of numerical masks as well as removing some low-S/N spectral orders from the cross-correlation; this did not significantly change the observed radial velocity variations. All these procedures were made for Run A using the version 3.6 of the HARPS-N DRS pipeline, which did not include any correction of the color effect. The DRS version 3.7, which includes a correction of the color effect, was available in 2014 and we used it for the data of Runs B to E.

\section{Empirical correction of the color effect on the radial velocities}

The radial velocities of $55 \mathrm{Cnc}$ were fitted with a Keplerian model taking the five planets of the system into account. For each run the fit was performed on the measurements outside of the transit of planet e, assumed to be on a circular orbit. Its transit epoch, transit duration, and period were taken from Dragomir et al. (2014), and the semi-amplitude of its radial velocity variations from Endl et al. (2012). Parameters for the other planets were also taken from Endl et al. (2012).

We observed a trend over each entire run in the radial velocity residuals from the Keplerian fit (see example for Run A in Fig. 1), which we interpret as being due to the color effect (Sect. 2). The CCF represents a mean profile of the thousands of stellar lines in the 69 HARPS-N spectral orders, whose Gaussian fit provides the radial velocity measurement. Because the flux color balance between the spectra varies during a run, this affects the relative contribution of each spectral order to the mean Doppler shift of the $\mathrm{CCF}$, and thus the measured radial velocity.

To characterize and quantify the chromatic variations, we defined a "color ratio" between the $\mathrm{S} / \mathrm{Ns}$ associated to the 69 spectral orders:

$$
C_{\mathrm{j} 1, \mathrm{j} 2}^{\mathrm{i} 1, \mathrm{i} 2}(\phi)=\frac{\sum_{i 1}^{i 2} S / N_{i}(\phi)}{\sum_{j 1}^{j 2} S / N_{j}(\phi)} .
$$

where $S / N_{\mathrm{k}}(\phi)$ represents the $\mathrm{S} / \mathrm{N}$ of spectral order $k$ ( $k$ varying from 0 to 68 , following the DRS pipeline orders numbering) at orbital phase $\phi$. The S/Ns at the numerator are summed between spectral orders $i 1$ and $i 2$ (included), and the $\mathrm{S} / \mathrm{Ns}$ at the denominator are summed between $j 1$ and $j 2$. We looked for the combination of spectral orders that gives the best correlation between the color ratio and the RV residuals to the Keplerian fit. For each combination we fitted the data outside of the transit with a polynomial regression, using the Bayesian information criterion (BIC) to prevent over-fitting with a high-order polynomial (Crossfield et al. 2012; Cowan et al. 2012). For each run we found a good correlation between the variations in the color ratio and those of the residuals to the Keplerian fit. As an example,
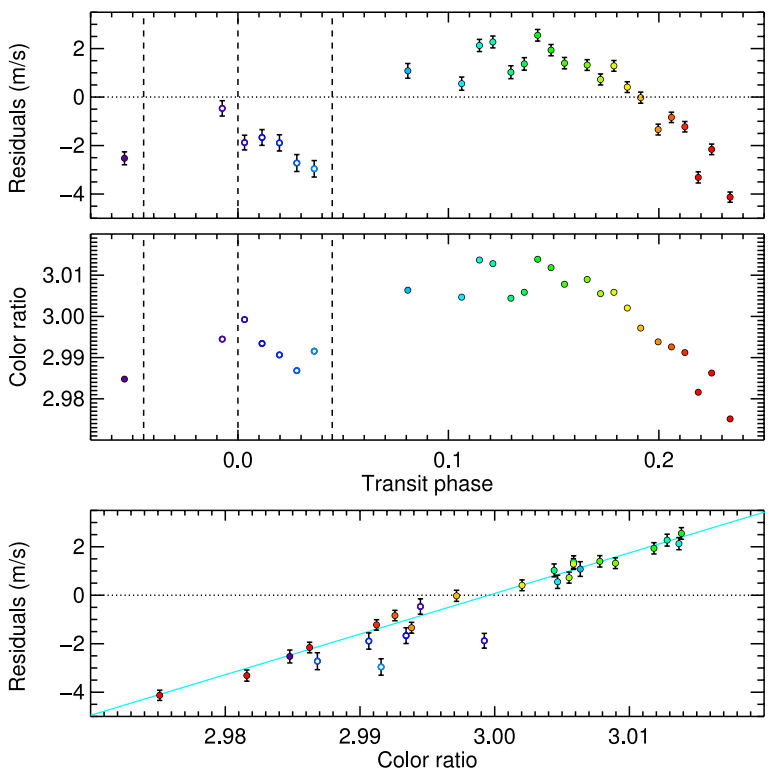

Fig. 1. Top: residuals from the Keplerian fit in Dataset A as a function of orbital phase. Vertical dashed lines show the times of mid-transit, first, and fourth contacts. The colors of the plotted circles indicate the orbital phases of each observation. Middle: color ratio $C_{32,32}^{28,30}$ as a function of orbital phase. There is a clear correlation with the RV residuals. The decrease in the color ratio at the end of the sequence is mainly due to the increase in the airmass. Bottom: linear relation between the residuals of the Keplerian fit and the color ratio. The fit is performed on the measurements outside of the transit (filled circles); those in the transit (empty circles) roughly follow the same trend.

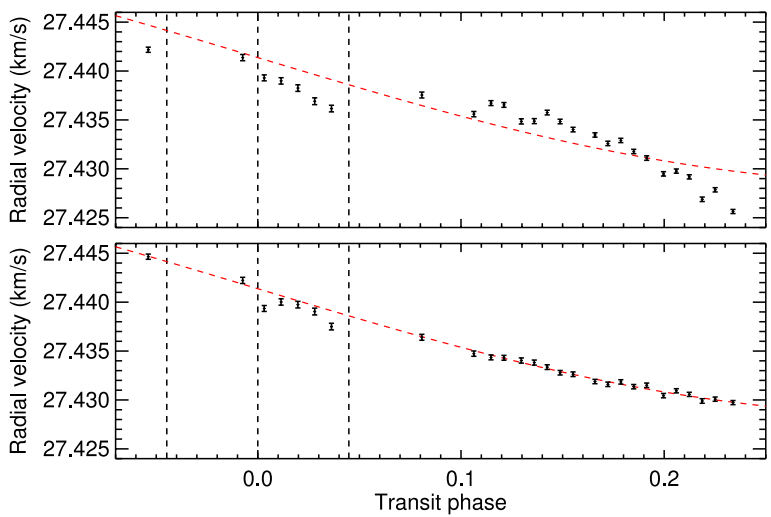

Fig. 2. Top: radial velocity measurements without any color-effect correction (black points) and their Keplerian fit (dashed red line) during Run A. Vertical dashed lines show the times of mid-transit, first, and fourth contacts. Bottom: after the empirical color-effect correction, radial velocity measurements outside of the transit are well-adjusted to the Keplerian fit, improving the out-of transit dispersion from $1.92 \mathrm{~m} / \mathrm{s}$ to $0.23 \mathrm{~m} / \mathrm{s}$.

Fig. 1 shows the similarities between the variations in these two quantities in the case of Run A, as well as their linear correlation. This allows us to apply an empirical correction of the color effect to the radial velocities, which improves their adjustment to the Keplerian curve (see, e.g., Run A in Fig. 2). The correlation is also shown in Fig. 3 in the case of Run E. As explained below, this run has the best transit observation.

Runs B to E include a DRS standard color-effect correction that was not available for Run A (see Sect. 2). We could thus apply our empirical correction on Datasets B to E, extracted without the DRS standard color-effect correction, and compare 
Table 2. Best parameters for the empirical correction of the color effect in each run.

\begin{tabular}{c|ccc|c|c}
\hline \hline & \multicolumn{3}{|c|}{ Empirical correction } & DRS standard correction & Without correction \\
\hline Run & $\begin{array}{c}\text { Color ratio } \\
{[i 1, i 2] /[j 1, j 2]}\end{array}$ & $\begin{array}{c}\text { Polynomial } \\
\text { degree }\end{array}$ & $\begin{array}{c}\text { Dispersion } \\
(\mathrm{m} / \mathrm{s})\end{array}$ & $\begin{array}{c}\text { Dispersion } \\
(\mathrm{m} / \mathrm{s})\end{array}$ & $\begin{array}{c}\text { Dispersion } \\
(\mathrm{m} / \mathrm{s})\end{array}$ \\
\hline $\mathrm{A}$ & {$[28,30] /[32,32]$} & 1 & 0.23 & - & 1.92 \\
$\mathrm{~B}$ & {$[15,17] /[12,12]$} & 3 & 0.32 & 0.73 & 2.57 \\
$\mathrm{C}$ & {$[14,16] /[13,13]$} & 1 & 0.67 & 0.95 & 1.11 \\
$\mathrm{D}$ & {$[6,7] /[4,5]$} & 1 & 0.60 & 1.52 & 1.39 \\
$\mathrm{E}$ & {$[21,21] /[28,28]$} & 1 & 0.28 & 0.43 & 1.86 \\
\hline
\end{tabular}

Notes. Dispersion is calculated outside of the transit on the residuals to the Keplerian fit. The corrections refer to the different color-effect corrections on the radial velocities.

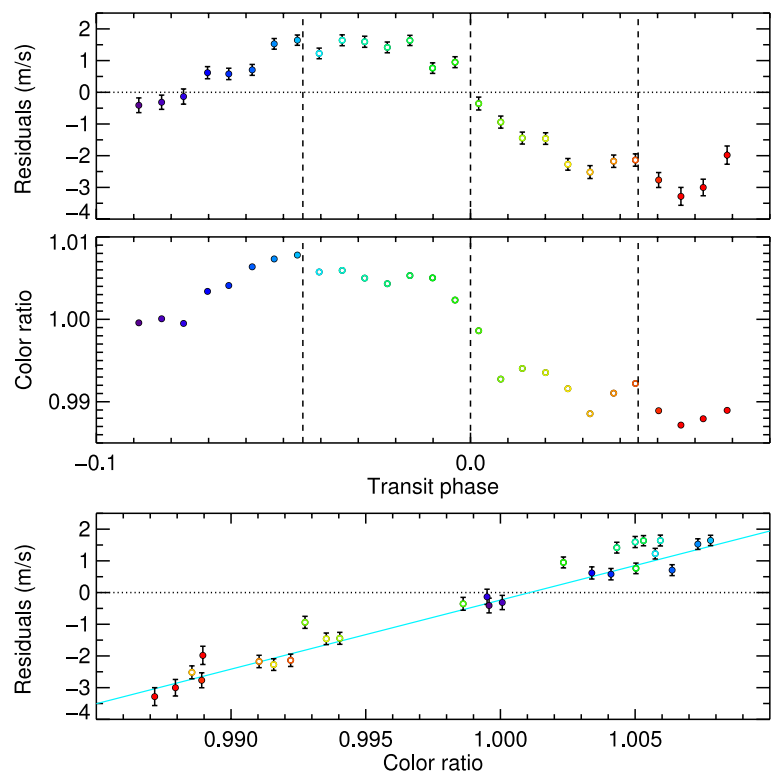

Fig. 3. Same plot as in Fig. 1 for Dataset E. Again, there is a linear correlation between the RV residuals and a color ratio, in this case $C_{28,28}^{21,21}$. As for Run A, the fit is performed on the measurements outside of the transit (filled circles), but those in the transit (empty circles) roughly follow the same trend.

the two different methods. Table 2 shows the dispersions of the residuals to the Keplerian fit outside of the transit for both color-effect corrections. The dispersion is always smaller in the case of our empirical correction, in some cases decreasing by more than a factor 2 . With the present observations of $55 \mathrm{Cnc}$ e, the empirical correction thus appears to give a better correction of the color effect than the DRS standard correction (Fig. 4 shows Dataset E reduced with both methods), and we adopt it hereafter.

We show in Table 2 the best-fit parameters for the empirical correction of each dataset. The best correlation between color ratio and RV residuals is always linear, except for Run B, which requires a third-order polynomial correction. Although this dataset has a high precision, it is apparently affected by additional systematics and shows oscillations with an amplitude up to several $\mathrm{dm} / \mathrm{s}$. We did not find any correlation between these oscillations and the color effect or any other parameter, and their origin is unclear. Datasets C and D have low S/Ns and still show a large, uncorrelated dispersion after correction. The last measurement of Dataset D had to be excluded to find an acceptable correlation between color ratio and RV residuals. Dataset A was obtained in different conditions than the other datasets, in particular with data during the transit secured in different conditions
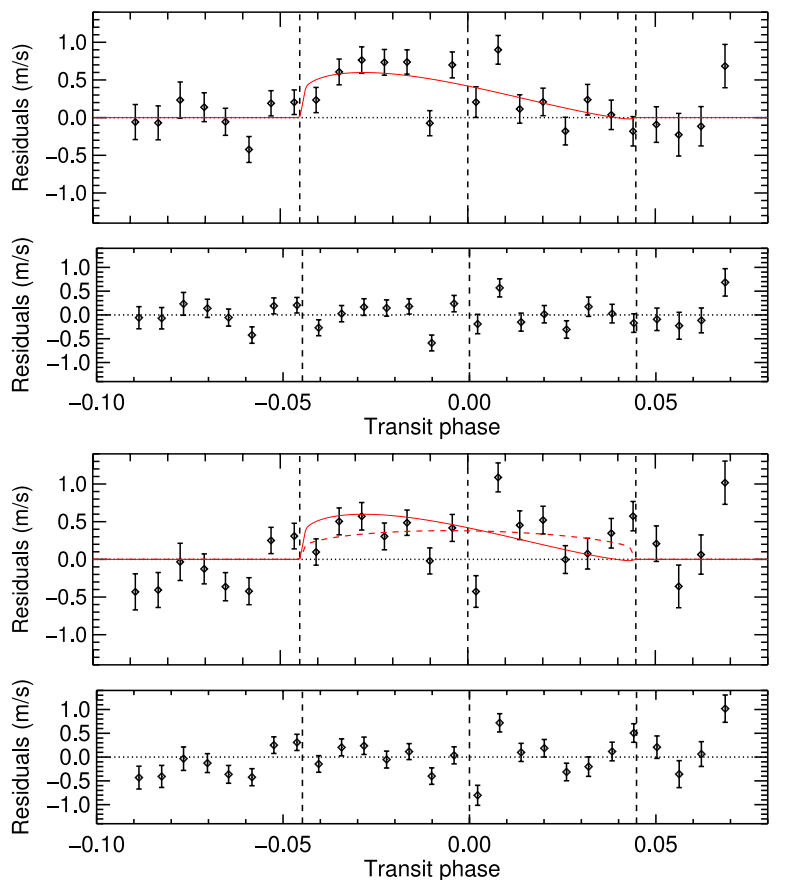

Fig. 4. Upper panels: residuals from the Keplerian fit in Dataset E (black diamonds), after the empirical color-effect correction; (top). The dispersion outside the transit is $28 \mathrm{~cm} / \mathrm{s}$. The solid red line shows the best fit of the Rossiter-McLaughlin anomaly with $\lambda=$ $72.4{ }_{-7.4}^{+9.0^{\circ}}$. The residuals to the Keplerian + RM fit over the entire sequence yield a dispersion of $28 \mathrm{~cm} / \mathrm{s}$ (bottom). Vertical dashed lines indicate the times of mid-transit, first, and fourth contacts. Lower panels: residuals from the Keplerian fit after the DRS standard color-effect correction (top). The dispersion outside the transit is $43 \mathrm{~cm} / \mathrm{s}$. The dashed red line shows the best fit of the Rossiter-McLaughlin anomaly with $\lambda=88.6_{-9.9}^{+9.3^{\circ}}$, in agreement with the best-fit obtained after the empirical correction (solid red line as in the upper panels). The DRS-corrected residuals to the Keplerian + RM fit over the entire sequence yield a dispersion of $39 \mathrm{~cm} / \mathrm{s}$ (bottom). Thus, both the Keplerian fit and the RM fit are better when using the empirical correction rather than the DRS correction.

than the reference ones outside the transit (see Sect. 2). Run E thus provides the best dataset, with a good sampling and a dispersion of the RV residuals after our empirical correction that is improved to the level where the Rossiter-McLaughlin anomaly can be detected for a super-Earth such as 55 Cnc e (Fig. 4). For these reasons, we first fit the Rossiter-McLaughlin anomaly in Dataset E only (Sect. 4) and then analyze the influence of the other datasets (Sect. 5). 
Table 3. Parameters for the Rossiter-McLaughlin analysis of $55 \mathrm{Cnc}$ e.

\begin{tabular}{lccc}
\hline \hline Parameter & Symbol & Value & Unit \\
\hline Fixed $^{\dagger}$ & & & \\
Transit epoch & $T_{0}$ & $2455962.0697_{-0.0018}^{+0.0017}$ & $\mathrm{BJD}$ \\
Transit duration & $t_{\mathrm{d}}$ & $0.0660_{-0.0028}^{+0.0035}$ & day \\
Orbital period & $P$ & $0.7365417_{-0.0000022}^{+0.000025}$ & day \\
Planet-to-star radii ratio & $R_{\mathrm{p}} / R_{*}$ & $0.01936_{-0.00075}^{+0.00079}$ & \\
Scaled semi-major axis & $a_{\mathrm{p}} / R_{*}$ & $3.523_{-0.040}^{+0.042}$ & \\
Orbital inclination & $i_{\mathrm{p}}$ & $85.4_{-2.1}^{+2.8}$ & $\mathrm{deg}$ \\
Stellar reflex velocity & $K$ & $6.3 \pm 0.21$ & $\mathrm{~m} / \mathrm{s}$ \\
\hline Measured & & & \\
Sky-projected obliquity & $\lambda$ & $72.4_{-11.5}^{+12.7}$ & $\mathrm{deg}$ \\
Projected stellar rotation velocity & $v \sin i_{*}$ & $3.3_{-0.9}^{+0.9}$ & $\mathrm{~km} \mathrm{~s}^{-1}$ \\
Systemic velocity & $\gamma$ & $27.40949 \pm 0.00008$ & $\mathrm{~km} \mathrm{~s}^{-1}$ \\
\hline
\end{tabular}

Notes. $^{(\dagger)}$ All parameters were taken from Dragomir et al. (2014), except for $K$ taken from Endl et al. (2012). (‡) Values are calculated in Sect. 4. See also Sect. 5.1 for the uncertainties.

\section{Detection of the Rossiter-McLaughlin anomaly and obliquity measurement}

After applying the empirical color-effect correction, radial velocities of Dataset $E$ were fitted in order to derive the sky-projected angle $\lambda$ between the planetary orbital axis and the stellar rotation axis. We applied the analytical approach developed by Ohta et al. (2005) to model the form of the RossiterMcLaughlin anomaly, which is described by ten parameters: the stellar limb-darkening linear coefficient $\epsilon$, the transit parameters $R_{\mathrm{p}} / R_{*}, a_{\mathrm{p}} / R_{*}$, and $i_{\mathrm{p}}$, the parameters of the circular orbit $\left(P, T_{0}\right.$, and $K$ ), the systemic radial velocity $\gamma$, the projected stellar rotation velocity $v \sin i_{*}$, and $\lambda$. We adopted a linear limb-darkening correction with $\epsilon=0.648$ (Dragomir et al. 2014). Parameters for the Keplerian fit are the same as in Sect. 3, and the additional transit parameters for planet e were taken from Dragomir et al. (2014; see Table 3).

We computed the $\chi^{2}$ of the fit on a grid scanning all possible values for $\lambda, v \sin i_{*}$, and $\gamma$. Once the minimum $\chi^{2}$ and corresponding best values for these parameters were obtained, we calculated their error bars from an analysis of the $\chi^{2}$ variation; that is, a given parameter is pegged at various trial values, and for each trial value we run an extra fit, allowing all the other parameters to vary freely. The $1 \sigma$ error bars for the pegged parameter are then obtained when its value yields a $\chi^{2}$ increase of 1 from the minimum (see, e.g., Hébrard et al. 2002). We detected the RM anomaly with $\lambda=72.4_{-6.1}^{+5.0^{\circ}}$ and $v \sin i_{*}=3.3 \pm 0.6 \mathrm{~km} \mathrm{~s}^{-1}$ (Fig. 4). The best fit provides a reduced $\chi^{2}$ of 2.2; to be conservative, we increased the error bars on the radial velocity measurements by a factor $\sqrt{2.2}$ to obtain a reduced $\chi^{2}$ of 1 . As a result, we adopt $\lambda=72.4_{-9.0}^{+7.4^{\circ}}$ and $v \sin i_{*}=3.3 \pm 0.9 \mathrm{~km} \mathrm{~s}^{-1}$. The derived $v \sin i_{*}$ agrees with the value $2.5 \pm 0.5 \mathrm{~km} \mathrm{~s}^{-1}$ obtained by Valenti \& Fischer (2005). The systemic radial velocity $\gamma$ was determined with a particularly high precision of $\pm 8 \mathrm{~cm} / \mathrm{s}$; however, it depends on the correlation mask, the spectral orders, and the color correction, so the actual barycentric stellar radial velocity is not as accurate. The dispersion of the residuals for the best fit $(28 \mathrm{~cm} / \mathrm{s})$ is similar to the estimated out-of-transit dispersion (Table 2). Results are summarized in Table 3.

We performed an F-test to evaluate the significance of the RM anomaly detection (see, e.g., Hébrard et al. 2011). We note that in our case the errors may not be normally distributed and

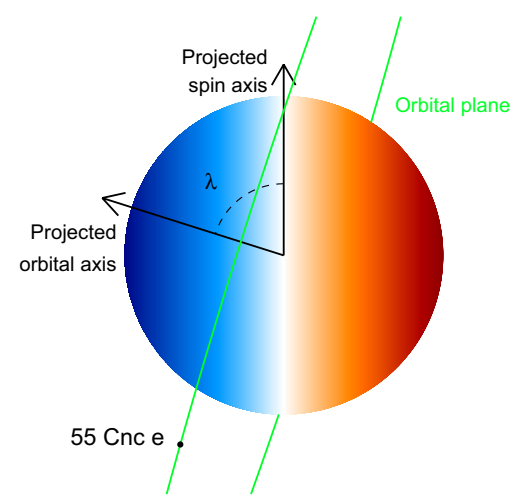

Fig. 5. View of $55 \mathrm{Cnc}$ along the line of sight. With the star rotation, the light emitted by the half of the stellar disk moving toward the observer is blueshifted, while the light from the other half that moves away is redshifted. During the transit, the small super-Earth $55 \mathrm{Cnc}$ e (shown as a black disk, to scale) mainly transits the blueshifted half of the stellar disk because of its high sky-projected obliquity $\lambda=72.4^{\circ}$.

independent, and thus the F-test is done as a rough estimate. Including the RM model in the fit improves the $\chi^{2}$ over the 27 measurements secured during Run E from 95.8 to 51.8, for two additional free parameters $\left(\lambda\right.$ and $\left.v \sin i_{*}\right)$. The statistical test indicates there is a probability $>90 \%$ that the $\chi^{2}$ improvement is due to the RM anomaly detected. We thus conclude that we detected the RM anomaly of $55 \mathrm{Cnc}$ e with $\lambda=72.4_{-9.0^{+7 .}}^{+0}$. Its orbit is prograde and highly misaligned, the planet transiting mainly the blueshifted regions of the stellar disk (Fig. 5).

\section{Validation tests}

Because of the small radius of the super-Earth $55 \mathrm{Cnc}$ e and the low rotation velocity of its star, the detection of its RM anomaly is challenging. In addition, we applied a new empirical method for correcting the color effect. Thus in this section we check the robustness of the detection of $55 \mathrm{Cnc}$ e RM anomaly presented in Sect. 4. To be conservative, all error bars on the free model parameters hereafter are scaled and enlarged to maintain a reduced $\chi^{2}$ of 1 . 


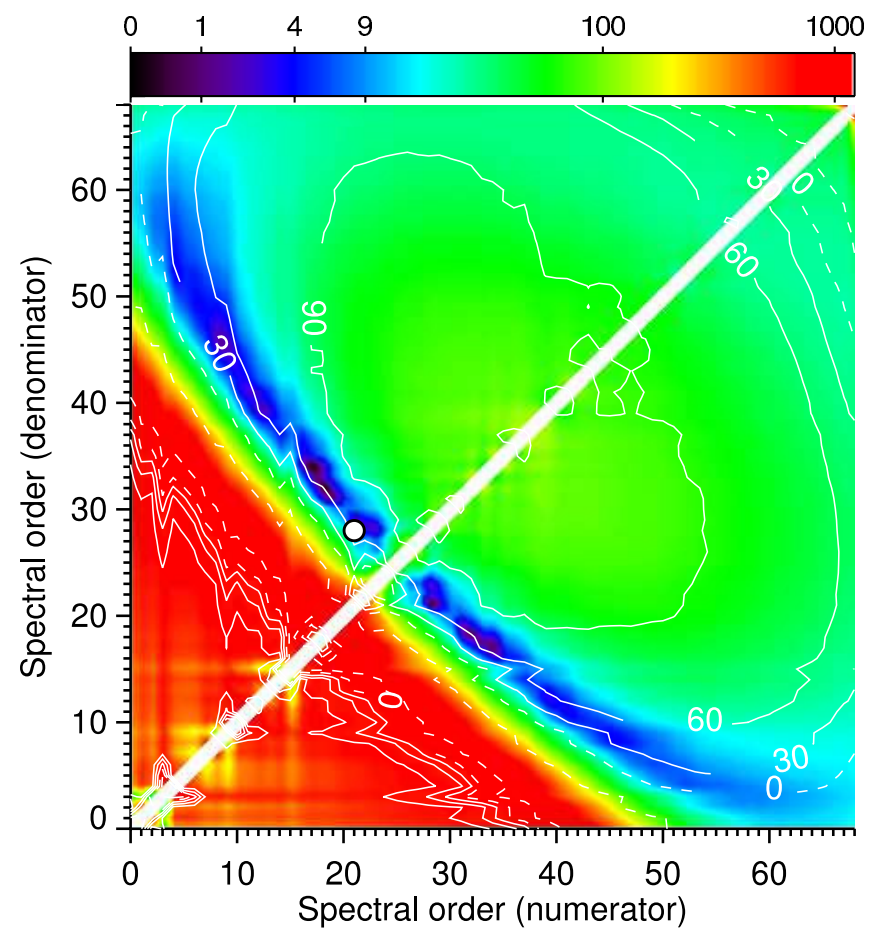

\subsection{Analysis of Run E}

In a first time we performed five kinds of tests on Dataset $\mathrm{E}$ alone.

\section{Fitting technique}

Minimizing the $\chi^{2}$ or the out-of-transit dispersion of the residuals, instead of the BIC, gives the same values for the spectral orders used in the empirical color-effect correction provided the polynomial degree is fixed to prevent overfitting.

Calculating the RM model by resampling each six-minute exposure by ten (e.g., Kipping 2010) has no influence on our results. This was expected because the modeled radial velocity variations during the exposure times are smaller than the error bars (see Fig. 4).

2. Ephemeris

The empirical color-effect correction is based on measurements outside of the $55 \mathrm{Cnc}$ e transit, to prevent removing its RM anomaly unintentionally. Thus we investigated how our results depend on $55 \mathrm{Cnc}$ e ephemeris, i.e., the midtransit $T_{0}$, the period $P$, and the transit duration $t_{\text {dur }}$. We quadratically propagated the errors on the mid-transit time taking the number of revolutions accomplished by $55 \mathrm{Cnc} \mathrm{e}$ between $T_{0}$ and Run E transit epoch into account.

Because we do not have enough measurements to constrain the transit mid-time and the period with high accuracy, we had to choose between the values derived by Dragomir et al. (2014); Gillon et al. (2012). We decided to adopt the values obtained by the former because they used long-time baseline MOST photometry of the $55 \mathrm{Cnc}$ sytem, with data obtained in 2012 in addition to the 2011 MOST data used by Gillon et al. (2012). This reduces the uncertainties on the mid-transit times of our runs, based on their measurement of $T_{0}$, and provides a higher precision value for the orbital period and planet to star radii ratio of $55 \mathrm{Cnc}$ e. Nonetheless, we also performed the RM anomaly fit using the ephemeris of Gillon et al. (2012). In this case $T_{0}(E)$, the mid-transit time of Run E, is about 11 min later, and the transit duration
Fig. 6. Dependence of the RM anomaly fit on the spectral orders used to compute the color-effect correction (Run E). Two different spectral orders must be used to quantify the color, which explains the white diagonal line where no fits can be done. White contours show the spin-orbit angles obtained for each color ratio (solid lines for positive values, dashed lines for negative values). The colorscale corresponds to the $\chi^{2}$ difference with respect to the best fit, obtained with the spectral orders 21 and 28 (white disk) and $\lambda=72.4^{\circ}$. Color ratios in the red part of the diagram show no significant correlation between the residuals of the Keplerian fit and the color ratio. Fits at less than about $3 \sigma$ from the best fit are found in the localized blue area.

about 8 min shorter, than with Dragomir et al. (2014). Three measurements switch between inside/outside the transit, and the color correction is thus different. We obtained $v \sin i_{*}=$ $3.6{ }_{-1.0}^{+0.7} \mathrm{~km} \mathrm{~s}^{-1}$, and $\lambda=102.9{ }_{-6.4}^{+11 .{ }^{\circ}}$ at $3 \sigma$ from the previous estimation in Sect. 4. It must be noted that the fit is of lower quality, with a reduced $\chi^{2}$ of 3.6 and a dispersion of the residuals to the RM fit of $36 \mathrm{~cm} / \mathrm{s}$ (instead of $\chi^{2}=2.2$ and a dispersion of $28 \mathrm{~cm} / \mathrm{s}$ ). Nonetheless we again detect the RM anomaly with a highly misaligned orbit, nearly polar.

We varied $T_{0}(E)$ within its $1 \sigma$ error bars using the ephemeris measured by Dragomir et al. (2014). Because of the large number of revolutions (1065) accomplished by $55 \mathrm{Cnc}$ e since the measure of $T_{0}$, the uncertainty on $T_{0}(E)$ is about $5.5 \mathrm{~min}$, roughly twice that of $T_{0}$. We also increased the transit duration by its upper $1 \sigma$ error bar $(\sim 5 \mathrm{~min})$. We found that using lower values for $T_{0}(E)$ has no significant impact on our results, whereas with higher values there are not enough measurements after the transit to properly correct for the color effect. This shows that enough measurements must be taken both before and after the transit for the color-effect correction to be efficient.

\section{Color-effect correction}

We fitted the RM anomaly to the data extracted with the DRS standard color-effect correction (Fig. 4). The anomaly is detected with $v \sin i_{*}=2.9 \pm 1.3 \mathrm{~km} \mathrm{~s}^{-1}$ and $\lambda=88.6_{-9.9}^{+9.3 \circ}$. This prograde, highly misaligned orbit is in good agreement with the RM anomaly detected after the empirical color-effect correction, although as expected the quality of the fit is lower with a reduced $\chi^{2}$ of 3.6 and a dispersion of the residuals to the RM fit of $39 \mathrm{~cm} / \mathrm{s}$.

We investigated how our results depend on the spectral orders used for the empirical color correction. We fixed a linear correction and calculated the best-fit parameters of the RM anomaly with all possible color ratios. To keep things simple, the color ratios are calculated with only two spectral orders (i.e. $i 1=i 2, j 1=j 2$ ). The results are shown in Fig. 6. As expected, the diagram is roughly symmetric, which shows that fits performed with a color ratio 
or its inverse (e.g., $C_{28,28}^{21,21}$ and $C_{21,21}^{28,28}$ ) have about the same quality and give similar values for $\lambda$. For most ratios, $\lambda$ is obtained with a high value between 50 and $110^{\circ}$ and stellar velocities between 0.5 and $5 \mathrm{~km} \mathrm{~s}^{-1}$. Only a specific range of spectral orders provides an acceptable adjustment to the data, all with $\lambda$-values around $70^{\circ}$. In this range, the best adjustments are obtained with a short separation between the spectral orders at the numerator and the denominator of the color ratio, as expected from our results for all datasets in Table 2.

\section{Model parameters}

The derived values remained within their uncertainties when we varied the limb-darkening coefficient $\epsilon$ between 0.1 and 0.9. This was expected from the precision of our measurements during the ingress and egress.

Increasing the eccentricity of the orbit up of 0.06 (e.g., Demory et al. 2012) and using other values for the semiamplitude of planet e (e.g., Nelson et al. 2014) does not change the shape of the Keplerian fit significantly during and around the transit, and has thus little infuence on our results. The same is true for the parameters of the outer planets.

Varying $R_{\mathrm{p}} / R_{*}$ and $a_{\mathrm{p}} / R_{*}$ within their small $1 \sigma$ error bars has no significant influence on our results. We used the values of Dragomir et al. (2014) for these two parameters, since the radius in particular is measured in the optical bandpass of MOST and is more appropriate to our analysis based on HARPS-N data than the radius measured in the infrared with Spitzer (Gillon et al. 2012).

We noted that the obliquity is sensitive to the inclination. While the quality of the fit remains unchanged, varying the inclination $i_{\mathrm{p}}$ between the $1 \sigma$ error bars obtained by Dragomir et al. (2014) results in uncertainties of $+10.3 /-7.2^{\circ}$ for $\lambda$. These uncertainties are calculated as the differences between the best-fit values in Sect. 4 and those obtained while varying $i_{\mathrm{p}}$. They are similar to the uncertainties derived in Sect. 4.

\section{Convective blueshift}

Because of the small amplitude of the measured RM anomaly $(\sim 60 \mathrm{~cm} / \mathrm{s})$, our interpretation of the radial velocity measurements may also be sensitive to the impact of the convective blueshift (CB) effect. We included the calculation of the $\mathrm{CB}$ radial velocity blueshift in our model, following the prescription of Shporer \& Brown (2011). Assuming a solar value for the local convective blueshift $(-300 \mathrm{~m} / \mathrm{s})$ and a linear limb-darkening law, we found that the CB effect has little influence on our results, with a maximum amplitude of about $4 \mathrm{~cm} / \mathrm{s}$ at the center of the transit which is well below the error bars on the radial velocity measurements. Since the distortion due to the $\mathrm{CB}$ effect increases with higher orbital inclinations, we performed the fit again while varying $i_{\mathrm{p}}$ between its $1 \sigma$ error bars. Even then, results were similar to those obtained when varying $i_{\mathrm{p}}$ without $\mathrm{CB}$ effect, the derived uncertainties varying by less than $1^{\circ}$ for $\lambda$ and $0.1 \mathrm{~km} \mathrm{~s}^{-1}$ for $v \sin i_{*}$ ). We also note that $55 \mathrm{Cnc}$ is a G8 star, and thus its local convective blueshift is likely lower than for the Sun.

To conclude, we took a conservative approach to calculating the final error bars on the obliquity. We adopted the quadratic sum of the uncertainties obtained in Sect. 4, and those obtained when the influence of the inclination was taken into account, to derive $\lambda=$ $72.4_{-11.5}^{+12.7^{\circ}}$. We caution that the value of $v \sin i_{*}$ may not be well constrained by our data, although this has no impact on the obliquity. While the analytic formula derived by Ohta et al. (2005) has been known to underestimate the velocity anomaly (e.g., Triaud et al. 2009; Hirano et al. 2010), it provides a good approximation when the stellar spin velocity is low enough. Increasing our bestfit value for $v \sin i_{*}$ by $10 \%$ (i.e., the systematic error in the case of HD 209458b, which rotates faster than 55 Cnc; Winn et al. 2005) was found to have no influence on the inferred obliquity. The value of $2.5 \pm 0.5 \mathrm{~km} \mathrm{~s}^{-1}$ obtained by Valenti \& Fischer (2005) may actually be a hint that our value for $v \sin i_{*}$ is overestimated. Assuming $v \sin i_{*}=2 \mathrm{~km} \mathrm{~s}^{-1}$, we obtained an obliquity of $62^{\circ}$ which remains within the derived uncertainties. Smaller $v \sin i_{*}$ values do not provide a good fit to the data, considering that the RM anomaly is detected with a significant amplitude.

\subsection{Analysis of all runs}

Although Runs A to D have lower qualities than Run E (Sect. 3), we checked their consistency with the RM anomaly detected on Dataset E. First we fit all datasets simultaneously, taking into account the five planets of the system in the Keplerian model. The radial velocity measurements after correction of the color effect are reported in Table 4 and displayed in Fig. 7. The errorweighted average of the Keplerian residuals over all runs clearly shows the RM anomaly detection despite the systematic errors (lower panel in Fig. 7). The results are within $1 \sigma$ of those obtained with Dataset E only with $\lambda=68.3 \pm 6.6^{\circ}$, but the dispersion of the RV residuals to the RM fit is much higher $(71 \mathrm{~cm} / \mathrm{s})$. We obtained similar results when fitting all runs simultaneously except Run E, with $\lambda=65.2 \pm 8.4^{\circ}$ and a dispersion of $77 \mathrm{~cm} / \mathrm{s}$.

Second we attempted to fit each dataset independently. Run A poorly samples the transit and was observed in two different modes; in addition the data secured during the transit were obtained in a different configuration than the reference data obtained after the transit. This makes Run A suspicious for the RM study, and indeed the fit did not succeed. As mentioned previously (Sect. 3), Run B shows radial velocity oscillations of unclear origin. This may be due to the presence of starspots or granulation on the stellar surface (e.g., Boisse et al. 2011; Dumusque et al. 2011) or an instrumental effect. Despite these perturbations, the empirical color-effect correction allows detecting the $\mathrm{RM}$ anomaly with $\lambda=77.1 \pm 7.3^{\circ}$ but a larger dispersion of the residuals than in Run E $(54 \mathrm{~cm} / \mathrm{s}$ instead of $28 \mathrm{~cm} / \mathrm{s})$. We obtain similar results with Dataset $\mathrm{C}$, although the presence of an outlier at orbital phase 0 results in an abnormally high value for $v \sin i_{*}$. Removing this outlier, we obtain $\lambda=65.9 \pm 15.2^{\circ}$ and a dispersion of $72 \mathrm{~cm} / \mathrm{s}$. Finally we performed an F-test for the RM anomaly in Run D in the same way as in Sect. 4, and found a $50 \%$ false positive probability due to the high noise in this dataset, indicating that the anomaly is likely not detected in this run.

We conclude that given their lower quality, Datasets A to D agrees with the RM anomaly detected in Run E.

\section{Discussion}

We report the detection of the Rossiter-McLaughlin anomaly of the super-Earth $55 \mathrm{Cnc}$ e, with a sky-projected obliquity $\lambda=72.4_{-11.5}^{+12.7^{\circ}}$. The planet is on a prograde and highly misaligned orbit, nearly polar. This detection is mainly based on one high-accuracy transit observed with HARPS-N, and thus more observations of the same quality as Run E are needed to confirm the detection. The super-Earth $55 \mathrm{Cnc}$ e is the smallest exoplanet for which the projected spin-orbit alignment has been 


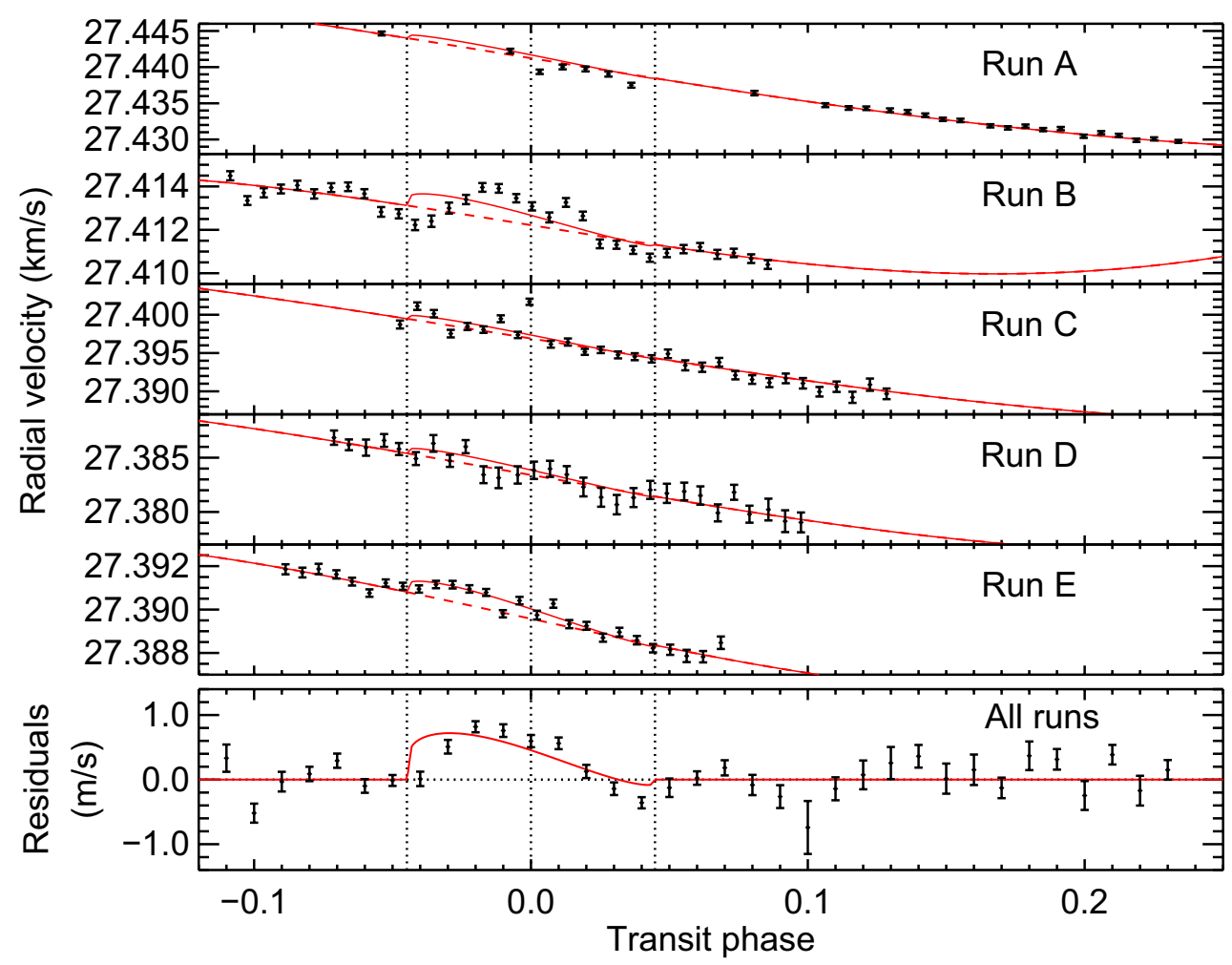

Fig. 7. Best model of the RM anomaly when fitting Datasets A to E simultaneously. Black points show radial velocity measurements as a function of the orbital phase (see Table 4), overlaid with the five-planet Keplerian fit ignoring the transit (dashed, red line), and the final fit including the model of the RM anomaly (solid, red line). Vertical dotted lines show the times of mid-transit, first, and fourth contacts. The simultaneous fit to the five runs provides similar results to the fit to Run E alone. The bottom panel shows the error-weighted average of the Keplerian residuals over all runs (residuals from the Keplerian fit are first calculated in each run and grouped in common phase bins of width 0.01). Although the combined residuals are dominated by systematic errors, the RM anomaly is clearly visible.

measured ${ }^{2}$, and is also the planet with the smallest RM anomaly amplitude detected $(\sim 0.6 \mathrm{~m} / \mathrm{s})$ below the Neptune-like exoplanet HAT-P-11 b $(1.5 \mathrm{~m} / \mathrm{s}$; Winn et al. $2010 \mathrm{~b})$ and Venus $(1 \mathrm{~m} / \mathrm{s}$; Molaro et al. 2013). We were able to detect the RM anomaly by devising an empirical color-effect correction for the chromatic variations known to affect radial velocity measurements. This correction is based on the S/Ns associated to HARPS-N spectral orders, and it may prove a useful tool for improving the accuracy of RV measurements from other stars or instruments. Indeed in the present study our empirical correction was found to improve the dispersion of the RV measurements with respect to the standard DRS correction, and with observing sequences of a few hours we detected the RM anomaly of $55 \mathrm{Cnc}$ e with high accuracy $(<30 \mathrm{~cm} / \mathrm{s})$.

The $55 \mathrm{Cnc}$ system is well approximated by a coplanar system (Kaib et al. 2011; Ehrenreich et al. 2012; Nelson et al. 2014), and thus all its planets are likely highly misaligned with the stellar spin axis. While most multiplanet systems have been found aligned with the stellar equator, this is the second occurrence of a highly misaligned one after Kepler-56 (Huber et al. 2013). This is a hint that large obliquities are not restricted to isolated hot-Jupiters as a consequence of a dynamical migration scenario. The high obliquity of $55 \mathrm{Cnc}$ e agrees with lower mass planets being either prograde and aligned, or strongly misaligned (Hébrard et al. 2010, 2011), although that trend was mainly seen

\footnotetext{
2 Stellar obliquities have been measured for the host stars of three smaller planets (Kepler-50b, $1.71 R_{\oplus}$; Kepler-65b, $1.42 R_{\oplus}$; Kepler-65d, $1.52 R_{\oplus}$ ) using asteroseismology (Chaplin et al. 2013), but this technique does not provide a direct measurement of the projected spin-orbit angle.
}

on isolated, Jupiter-mass planets. It is also a new exception to the apparent trend that misaligned planets tend to orbit hot stars (Winn et al. 2010a; the effective temperature of 55 Cnc derived by von Braun et al. 2011 is $\left.T_{\text {eff }}=5196 \mathrm{~K}\right)$. That tidal interactions did not align the system (Barker \& Ogilvie 2009) during its long lifetime (10.2 Gy; von Braun et al. 2011) may be due to the low mass of its star, the low mass of its closest companion $55 \mathrm{Cnc}$ e, and the complex dynamical interactions within this compact multiple system (Nelson et al. 2014). The particularity of the 55 Cnc and Kepler-56 systems may be the presence of a wide-orbit companion. Although such companions may be present in other multiple systems, none have been detected. If the companion is initially inclined with respect to the protoplanetary disk, or with the inner planets around the primary star, it may misalign their orbital planes while preserving their coplanarity (e.g., Batygin 2012; Kaib et al. 2011). Kaib et al. (2011) investigated this scenario in the case of the $55 \mathrm{Cnc}$ system, whose stellar companion $55 \mathrm{Cnc} \mathrm{B}$ was detected at a projected distance of 1065 AU (Mugrauer et al. 2006). With a semi-major axis lower than about 4000 au the gravitational influence of $55 \mathrm{Cnc} \mathrm{B}$ is strong enough to significantly alter the alignment of the system, provided the star is on a highly eccentric orbit $(e \gtrsim 0.95$; Boué \& Fabrycky 2014). Kaib et al. (2011) predict a true obliquity of $\sim 65^{\circ}$, which is remarkably consistent with the sky-projected obliquity of $72.4_{-11.5}^{+12.7^{\circ}}$ we derived, and indicate that the rotation axis of $55 \mathrm{Cnc} \mathrm{A}$ is probably not inclined much toward the line of sight.

Acknowledgements. We deeply thank the referee T. Hirano for his thoughtful comments. We would also like to thank J.-M. Almenara, F. Bouchy, M. Deleuil, R. F. Díaz, A. Lecavelier des Étangs, G. Montagnier, C. Moutou, 
and A. Santerne for their help and advice. This publication is based on observations collected with the the HARPS-N spectrograph on the 3.58-m Italian Telescopio Nazionale Galileo (TNG) operated on the island of La Palma by the Fundación Galileo Galilei of the INAF (Instituto Nazionale di Astrofisica) at the Spanish Observatorio del Roque de los Muchachos of the Instituto de Astrofisica de Canarias (programs OPT12B_13, OPT13B_30, and OPT14A_34 from OPTICON common time allocation process for EC supported transnational access to European telescopes). We thank the TNG staff for support. The authors acknowledge the support of the French Agence Nationale de la Recherche (ANR), under program ANR-12-BS05-0012 "Exo-Atmos".

\section{References}

Albrecht, S., Winn, J. N., Johnson, J. A., et al. 2012, ApJ, 757, 18 Albrecht, S., Winn, J. N., Marcy, G. W., et al. 2013, ApJ, 771, 11 Baranne, A., Queloz, D., Mayor, M., et al. 1996, A\&AS, 119, 373 Barker, A. J., \& Ogilvie, G. I. 2009, MNRAS, 395, 2268

Batygin, K. 2012, Nature, 491, 418

Boisse, I., Bouchy, F., Hébrard, G., et al. 2011, A\&A, 528, A4

Boué, G., \& Fabrycky, D. C. 2014, ApJ, 789, 111

Cébron, D., Moutou, C., Le Bars, M., Le Gal, P., \& Fares, R. 2011 [arXiv: 1101.4531]

Chaplin, W. J., Sanchis-Ojeda, R., Campante, T. L., et al. 2013, ApJ, 766, 101

Cosentino, R., Lovis, C., Pepe, F., et al. 2012, in SPIE Conf. Ser., 8446

Cowan, N. B., Machalek, P., Croll, B., et al. 2012, ApJ, 747, 82

Crida, A., \& Batygin, K. 2014, A\&A, 567, A42

Crossfield, I. J. M., Knutson, H., Fortney, J., et al. 2012, ApJ, 752, 81

Dawson, R. I., \& Fabrycky, D. C. 2010, ApJ, 722, 937

Demory, B.-O., Gillon, M., Deming, D., et al. 2011, A\&A, 533, A114

Demory, B.-O., Gillon, M., Seager, S., et al. 2012, ApJ, 751, L28

Dragomir, D., Matthews, J. M., Winn, J. N., \& Rowe, J. F. 2014, in IAU Symp.

293, ed. N. Haghighipour, 52

Dumusque, X., Santos, N. C., Udry, S., Lovis, C., \& Bonfils, X. 2011, A\&A, 527, A 82

Ehrenreich, D., Bourrier, V., Bonfils, X., et al. 2012, A\&A, 547, A18

Endl, M., Robertson, P., Cochran, W. D., et al. 2012, ApJ, 759, 19

Fabrycky, D., \& Tremaine, S. 2007, ApJ, 669, 1298

Fischer, D. A., Marcy, G. W., Butler, R. P., et al. 2008, ApJ, 675, 790

Gillon, M., Demory, B.-O., Benneke, B., et al. 2012, A\&A, 539, A28

Guillochon, J., Ramirez-Ruiz, E., \& Lin, D. 2011, ApJ, 732, 74

Hébrard, G., Lemoine, M., Vidal-Madjar, A., et al. 2002, ApJS, 140, 103
Hébrard, G., Bouchy, F., Pont, F., et al. 2008, A\&A, 488, 763

Hébrard, G., Désert, J.-M., Díaz, R. F., et al. 2010, A\&A, 516, A95

Hébrard, G., Ehrenreich, D., Bouchy, F., et al. 2011, A\&A, 527, L11

Hirano, T., Suto, Y., Taruya, A., et al. 2010, ApJ, 709, 458

Hirano, T., Narita, N., Sato, B., et al. 2011, PASJ, 63, L57

Hirano, T., Narita, N., Sato, B., et al. 2012, ApJ, 759, L36

Hirano, T., Sanchis-Ojeda, R., Takeda, Y., et al. 2014, ApJ, 783, 9

Holt, J. R. 1893, A\&A, 12, 646

Huber, D., Carter, J. A., Barbieri, M., et al. 2013, Science, 342, 331

Kaib, N. A., Raymond, S. N., \& Duncan, M. J. 2011, ApJ, 742, L24

Kipping, D. M. 2010, MNRAS, 408, 1758

Lai, D., Foucart, F., \& Lin, D. N. C. 2011, MNRAS, 412, 2790

Loeillet, B., Shporer, A., Bouchy, F., et al. 2008, A\&A, 481, 529

McLaughlin, D. B. 1924, ApJ, 60, 22

Molaro, P., Monaco, L., Barbieri, M., \& Zaggia, S. 2013, MNRAS, 429, L79

Moutou, C., Hébrard, G., Bouchy, F., et al. 2009, A\&A, 498, L5

Mugrauer, M., Neuhäuser, R., Mazeh, T., et al. 2006, Astron. Nachr., 327, 321

Narita, N., Hirano, T., Sanchis-Ojeda, R., et al. 2010, PASJ, 62, L61

Nelson, B. E., Ford, E. B., Wright, J. T., et al. 2014, MNRAS [arXiv: 1402.6343]

Ohta, Y., Taruya, A., \& Suto, Y. 2005, ApJ, 622, 1118

Pepe, F., Mayor, M., Galland, F., et al. 2002, A\&A, 388, 632

Pont, F., Hébrard, G., Irwin, J. M., et al. 2009, A\&A, 502, 695

Queloz, D., Eggenberger, A., Mayor, M., et al. 2000, A\&A, 359, L13

Rossiter, R. A. 1924, ApJ, 60, 15

Sanchis-Ojeda, R., Fabrycky, D. C., Winn, J. N., et al. 2012, Nature, 487, 449

Shporer, A., \& Brown, T. 2011, ApJ, 733, 30

Teyssandier, J., Terquem, C., \& Papaloizou, J. C. B. 2013, MNRAS, 428, 658

Triaud, A. H. M. J., Queloz, D., Bouchy, F., et al. 2009, A\&A, 506, 377

Triaud, A. H. M. J., Collier Cameron, A., Queloz, D., et al. 2010, A\&A, 524, A25

Valenti, J. A., \& Fischer, D. A. 2005, ApJS, 159, 141

Van Eylen, V., Lund, M. N., Silva Aguirre, V., et al. 2014, ApJ, 782, 14

von Braun, K., Boyajian, T. S., ten Brummelaar, T. A., et al. 2011, ApJ, 740, 49

Walkowicz, L. M., \& Basri, G. S. 2013, MNRAS, 436, 1883

Winn, J. N., Noyes, R. W., Holman, M. J., et al. 2005, ApJ, 631, 1215

Winn, J. N., Howard, A. W., Johnson, J. A., et al. 2009a, ApJ, 703, 2091

Winn, J. N., Johnson, J. A., Albrecht, S., et al. 2009b, ApJ, 703, L99

Winn, J. N., Johnson, J. A., Fabrycky, D., et al. 2009c, ApJ, 700, 302

Winn, J. N., Fabrycky, D., Albrecht, S., \& Johnson, J. A. 2010a, ApJ, 718, L145

Winn, J. N., Johnson, J. A., Howard, A. W., et al. 2010b, ApJ, 723, L223

Winn, J. N., Matthews, J. M., Dawson, R. I., et al. 2011, ApJ, 737, L18 
Table 4. Radial velocities of 55 Cnc, after the empirical color-effect Table 4. continued. correction (plotted in Fig. 7).

\begin{tabular}{|c|c|c|c|}
\hline & $\begin{array}{c}\text { BJD } \\
-2400000\end{array}$ & $\begin{array}{c}\mathrm{RV} \\
\left(\mathrm{km} \mathrm{s}^{-1}\right)\end{array}$ & $\begin{array}{c} \pm 1 \sigma \\
\left(\mathrm{km} \mathrm{s}^{-1}\right)\end{array}$ \\
\hline \multirow[t]{27}{*}{ Run A } & 56287.5814 & 27.44465 & 0.00027 \\
\hline & 56287.6156 & 27.44222 & 0.00032 \\
\hline & 56287.6235 & 27.43935 & 0.00030 \\
\hline & 56287.6296 & 27.44002 & 0.00033 \\
\hline & 56287.6357 & 27.43974 & 0.00034 \\
\hline & 56287.6417 & 27.43904 & 0.00035 \\
\hline & 56287.6478 & 27.43750 & 0.00034 \\
\hline & 56287.6806 & 27.43641 & 0.00030 \\
\hline & 56287.6994 & 27.43474 & 0.00027 \\
\hline & 56287.7057 & 27.43435 & 0.00025 \\
\hline & 56287.7103 & 27.43431 & 0.00024 \\
\hline & 56287.7167 & 27.43403 & 0.00027 \\
\hline & 56287.7213 & 27.43382 & 0.00026 \\
\hline & 56287.7260 & 27.43335 & 0.00024 \\
\hline & 56287.7307 & 27.43278 & 0.00023 \\
\hline & 56287.7354 & 27.43263 & 0.00024 \\
\hline & 56287.7433 & 27.43188 & 0.00022 \\
\hline & 56287.7480 & 27.43160 & 0.00023 \\
\hline & 56287.7527 & 27.43185 & 0.00022 \\
\hline & 56287.7574 & 27.43136 & 0.00022 \\
\hline & 56287.7621 & 27.43150 & 0.00023 \\
\hline & 56287.7682 & 27.43044 & 0.00022 \\
\hline & 56287.7728 & 27.43094 & 0.00021 \\
\hline & 56287.7775 & 27.43057 & 0.00022 \\
\hline & 56287.7822 & 27.42989 & 0.00023 \\
\hline & 56287.7869 & 27.43008 & 0.00022 \\
\hline & 56287.7934 & 27.42973 & 0.00021 \\
\hline \multirow[t]{33}{*}{ Run B } & 56659.4947 & 27.41449 & 0.00021 \\
\hline & 56659.4992 & 27.41335 & 0.00020 \\
\hline & 56659.5036 & 27.41371 & 0.00021 \\
\hline & 56659.5082 & 27.41388 & 0.00020 \\
\hline & 56659.5125 & 27.41405 & 0.00021 \\
\hline & 56659.5170 & 27.41366 & 0.00021 \\
\hline & 56659.5216 & 27.41395 & 0.00021 \\
\hline & 56659.5260 & 27.41399 & 0.00020 \\
\hline & 56659.5304 & 27.41367 & 0.00021 \\
\hline & 56659.5348 & 27.41283 & 0.00022 \\
\hline & 56659.5395 & 27.41274 & 0.00021 \\
\hline & 56659.5439 & 27.41223 & 0.00024 \\
\hline & 56659.5482 & 27.41240 & 0.00026 \\
\hline & 56659.5529 & 27.41300 & 0.00025 \\
\hline & 56659.5574 & 27.41340 & 0.00021 \\
\hline & 56659.5618 & 27.41396 & 0.00020 \\
\hline & 56659.5661 & 27.41391 & 0.00020 \\
\hline & 56659.5708 & 27.41346 & 0.00019 \\
\hline & 56659.5750 & 27.41308 & 0.00019 \\
\hline & 56659.5796 & 27.41257 & 0.00023 \\
\hline & 56659.5840 & 27.41327 & 0.00020 \\
\hline & 56659.5885 & 27.41264 & 0.00020 \\
\hline & 56659.5930 & 27.41136 & 0.00020 \\
\hline & 56659.5975 & 27.41132 & 0.00019 \\
\hline & 56659.6019 & 27.41107 & 0.00018 \\
\hline & 56659.6063 & 27.41072 & 0.00019 \\
\hline & 56659.6108 & 27.41093 & 0.00019 \\
\hline & 56659.6153 & 27.41111 & 0.00019 \\
\hline & 56659.6197 & 27.41121 & 0.00018 \\
\hline & 56659.6243 & 27.41087 & 0.00021 \\
\hline & 56659.6287 & 27.41094 & 0.00019 \\
\hline & 56659.6333 & 27.41067 & 0.00020 \\
\hline & 56659.6377 & 27.41040 & 0.00020 \\
\hline
\end{tabular}

\begin{tabular}{|c|c|c|c|}
\hline & $\begin{array}{c}\text { BJD } \\
-2400000\end{array}$ & $\begin{array}{c}\mathrm{RV} \\
\left(\mathrm{km} \mathrm{s}^{-1}\right)\end{array}$ & $\begin{array}{c} \pm 1 \sigma \\
\left(\mathrm{km} \mathrm{s}^{-1}\right)\end{array}$ \\
\hline \multirow[t]{30}{*}{ Run C } & 56684.5823 & 27.39872 & 0.00052 \\
\hline & 56684.5869 & 27.40112 & 0.00049 \\
\hline & 56684.5913 & 27.40014 & 0.00048 \\
\hline & 56684.5958 & 27.39753 & 0.00049 \\
\hline & 56684.6002 & 27.39847 & 0.00047 \\
\hline & 56684.6045 & 27.39806 & 0.00042 \\
\hline & 56684.6091 & 27.39947 & 0.00045 \\
\hline & 56684.6136 & 27.39736 & 0.00041 \\
\hline & 56684.6167 & 27.40169 & 0.00041 \\
\hline & 56684.6224 & 27.39615 & 0.00045 \\
\hline & 56684.6269 & 27.39644 & 0.00047 \\
\hline & 56684.6314 & 27.39516 & 0.00040 \\
\hline & 56684.6357 & 27.39541 & 0.00042 \\
\hline & 56684.6403 & 27.39476 & 0.00045 \\
\hline & 56684.6447 & 27.39452 & 0.00045 \\
\hline & 56684.6492 & 27.39424 & 0.00048 \\
\hline & 56684.6536 & 27.39490 & 0.00057 \\
\hline & 56684.6582 & 27.39339 & 0.00065 \\
\hline & 56684.6627 & 27.39314 & 0.00060 \\
\hline & 56684.6672 & 27.39379 & 0.00057 \\
\hline & 56684.6715 & 27.39210 & 0.00054 \\
\hline & 56684.6760 & 27.39154 & 0.00057 \\
\hline & 56684.6805 & 27.39113 & 0.00059 \\
\hline & 56684.6849 & 27.39169 & 0.00063 \\
\hline & 56684.6895 & 27.39105 & 0.00068 \\
\hline & 56684.6939 & 27.38994 & 0.00062 \\
\hline & 56684.6984 & 27.39060 & 0.00068 \\
\hline & 56684.7027 & 27.38922 & 0.00073 \\
\hline & 56684.7073 & 27.39087 & 0.00080 \\
\hline & 56684.7117 & 27.38967 & 0.00069 \\
\hline \multirow[t]{30}{*}{ Run D } & 56715.4995 & 27.38685 & 0.00066 \\
\hline & 56715.5034 & 27.38619 & 0.00050 \\
\hline & 56715.5080 & 27.38593 & 0.00075 \\
\hline & 56715.5128 & 27.38659 & 0.00059 \\
\hline & 56715.5167 & 27.38580 & 0.00056 \\
\hline & 56715.5213 & 27.38492 & 0.00059 \\
\hline & 56715.5259 & 27.38632 & 0.00077 \\
\hline & 56715.5303 & 27.38471 & 0.00056 \\
\hline & 56715.5345 & 27.38602 & 0.00061 \\
\hline & 56715.5392 & 27.38343 & 0.00078 \\
\hline & 56715.5433 & 27.38314 & 0.00095 \\
\hline & 56715.5483 & 27.38341 & 0.00080 \\
\hline & 56715.5527 & 27.38384 & 0.00080 \\
\hline & 56715.5569 & 27.38398 & 0.00074 \\
\hline & 56715.5614 & 27.38345 & 0.00078 \\
\hline & 56715.5658 & 27.38230 & 0.00086 \\
\hline & 56715.5705 & 27.38135 & 0.00087 \\
\hline & 56715.5747 & 27.38067 & 0.00089 \\
\hline & 56715.5792 & 27.38133 & 0.00088 \\
\hline & 56715.5836 & 27.38203 & 0.00083 \\
\hline & 56715.5880 & 27.38171 & 0.00089 \\
\hline & 56715.5926 & 27.38189 & 0.00081 \\
\hline & 56715.5969 & 27.38151 & 0.00084 \\
\hline & 56715.6016 & 27.37991 & 0.00078 \\
\hline & 56715.6059 & 27.38181 & 0.00069 \\
\hline & 56715.6100 & 27.37979 & 0.00078 \\
\hline & 56715.6150 & 27.38022 & 0.00100 \\
\hline & 56715.6195 & 27.37915 & 0.00101 \\
\hline & 56715.6237 & 27.37904 & 0.00091 \\
\hline & 56715.6283 & 27.37524 & 0.00100 \\
\hline
\end{tabular}


V. Bourrier \& G. Hébrard: Detecting the spin-orbit misalignment of the super-Earth $55 \mathrm{Cnc}$ e

Table 4. continued.

\begin{tabular}{cccc}
\hline \hline & $\begin{array}{c}\text { BJD } \\
-2400000\end{array}$ & $\begin{array}{c}\text { RV } \\
\left(\mathrm{km} \mathrm{s}^{-1}\right)\end{array}$ & $\begin{array}{c} \pm 1 \sigma \\
\left(\mathrm{km} \mathrm{s}^{-1}\right)\end{array}$ \\
\hline Run E & 56746.4213 & 27.39186 & 0.00023 \\
& 56746.4258 & 27.39171 & 0.00023 \\
& 56746.4301 & 27.39187 & 0.00024 \\
56746.4348 & 27.39162 & 0.00019 \\
56746.4390 & 27.39129 & 0.00018 \\
56746.4436 & 27.39076 & 0.00017 \\
56746.4480 & 27.39122 & 0.00017 \\
56746.4525 & 27.39107 & 0.00016 \\
56746.4568 & 27.39095 & 0.00017 \\
56746.4613 & 27.39115 & 0.00017 \\
56746.4658 & 27.39115 & 0.00018 \\
56746.4702 & 27.39095 & 0.00017 \\
56746.4747 & 27.39079 & 0.00016 \\
56746.4791 & 27.38981 & 0.00017 \\
56746.4836 & 27.39041 & 0.00017 \\
56746.4882 & 27.38974 & 0.00020 \\
56746.4926 & 27.39027 & 0.00019 \\
56746.4968 & 27.38933 & 0.00019 \\
56746.5014 & 27.38925 & 0.00018 \\
56746.5058 & 27.38870 & 0.00018 \\
56746.5101 & 27.38896 & 0.00020 \\
56746.5148 & 27.38859 & 0.00020 \\
56746.5191 & 27.38822 & 0.00020 \\
56746.5236 & 27.38815 & 0.00024 \\
56746.5280 & 27.38786 & 0.00028 \\
56746.5324 & 27.38783 & 0.00026 \\
56746.5371 & 27.38847 & 0.00029 \\
\hline & &
\end{tabular}

